\author{
Classification \\ Physics Abstracts \\ 0.660
}

\title{
ÉTUDE D'UN DISJONCTEUR RAPIDE POUR COURANTS INTENSES
}

\author{
A. DELMAS, C. RIOUX et F. RIOUX-DAMIDAU \\ Groupe Electrotechnique et Fusion Contrôlée, \\ Laboratoire de Physique des Plasmas, Bât. 214, 91405 Orsay, France
}

(Reçu le 7 février 1975, accepté le 11 mars 1975)

\begin{abstract}
Résumé. - Le disjoncteur présenté dans cet article est capable de couper un courant de $10 \mathrm{kA}$ en $1 \mu$ s et de supporter une surtension de l'ordre de $20 \mathrm{kV}$ pour une distance d'isolement de $3 \mathrm{~mm}$. Ce disjoncteur est du type à rupture mécanique : le remplacement brutal d'un morceau de conducteur par un isolant est assuré par la surpression engendrée par l'explosion d'un mélange stœchiométrique d'hydrogène et d'oxygène. Le courant est alors dérivé sur une impédance variable (fusible) qui assure, soit le transfert d'énergie sur la charge, soit l'ouverture du circuit.
\end{abstract}

\begin{abstract}
The circuit breaker presented here is capable of interrupting a $10 \mathrm{kA}$ current within $1 \mu \mathrm{s}$ and then sustain an over voltage of the order of $20 \mathrm{kV}$ for a $3 \mathrm{~mm}$ insulation width. The construction is of the mechanical rupture type in which the replacement of a piece of conductor by an insulator is ensured by means of the explosion of a stoichiometric mixture of hydrogen and oxygen. The current is then transfered into a fuse which finally transfers the energy to the load or opens the circuit.
\end{abstract}

1. Introduction. - Jusqu'à maintenant, les sources délivrant de fortes énergies en des temps très courts nécessaires à de nombreuses expériences ont été constituées par des bancs de condensateurs. Cependant, la demande d'une énergie nominale de plus en plus élevée conduit à se tourner vers de nouvelles sources utilisant le stockage inductif. Pour délivrer en quelques microsecondes sur une charge l'énergie stockée dans une bobine pendant un temps relativement long (par exemple $1 \mathrm{~s}$ ), il est alors nécessaire de disposer de disjoncteurs rapides pour courants intenses. Le problème de la réalisation de ces disjoncteurs est un des problèmes clés conditionnant les performances des sources rapides et qui n'est pas encore résolu. Nous avons déjà montré dans une étude théorique précédente [1] qu'il était préférable d'intercaler entre la bobine de stockage et l'interrupteur proprement dit un système de transfert inductif à haut rendement [2]; le temps pendant lequel le disjoncteur doit laisser passer le courant avant d'être ouvert est alors réduit à $10^{-4} \mathrm{~s}$ environ.

Il est par ailleurs indiqué d'assurer la commutation du courant sur une charge d'impédance variable [3]. Ceci est rendu possible par la mise en parallèle d'un fil explosant. Enfin nous avons déterminé que l'interrupteur devait être du type à rupture mécanique de conducteur (cuivre ou aluminium). Nous présentons ici un disjoncteur de ce type, dans lequel la pression assurant la rupture du conducteur est fournie par l'explosion d'un mélange $2 \mathrm{H}_{2}+\mathrm{O}_{2}$ sous pression. Associé à un fil explosant, il a permis de couper des courants de l'ordre de $10^{4} \mathrm{~A}$ en $1 \mu$ s et de tenir une surtension de $20 \mathrm{kV}$.

2. Description du montage expérimental. - 2.1 CHOIX DE LA STRUCTURE. - Dans les disjoncteurs à rupture mécanique du conducteur, on applique brutalement une surpression sur l'isolant préalablement accolé au conducteur, ce qui provoque sa rupture. L'isolant libéré souffle l'arc qui apparaît au niveau de la coupure et dégage le morceau cassé. Celui-ci peut encore être projeté dans de l'isolant, ce qui permet une extinction plus rapide de l'arc.

Dans des expériences préliminaires, la surpression avait été engendrée par le choc d'un marteau tombant directement sur l'isolant (graisse [4]). La synchronisation d'un tel système étant difficile et le but étant de faire fonctionner plusieurs interrupteurs en parallèle, nous avons préféré utiliser l'onde de choc créée par la mise à feu d'un explosif.

Afin d'éviter une détérioration rapide et coûteuse des structures du disjoncteur, nous avons utilisé non pas un explosif solide mais le mélange $2 \mathrm{H}_{2}+\mathrm{O}_{2}$ dont l'effet brisant est beaucoup plus modéré. En effet, si 
nous assimilons la vapeur d'eau formée par l'explosion du mélange $2 \mathrm{H}_{2}+\mathrm{O}_{2}$ à un gaz parfait et si nous appelons $\gamma$ le rapport des chaleurs spécifiques à pression et volume constants pour cette vapeur, la relation qui lie sa pression $P$ lorsque l'équilibre thermodynamique est rétabli à la pression initiale $P_{1}$ du mélange s'écrit :

$$
\frac{P}{\gamma-1}=\frac{Q}{V_{1}}=K P_{1}
$$

$Q$ est la chaleur dégagée par la combustion du mélange de volume $V_{1} ; Q / V_{1}$ représente donc la densité d'énergie libérée qui est proportionnelle à la pression initiale $P_{1}$, le coefficient $K$ valant 71,5. Aux températures voisines de la température d'explosion, $\gamma$ est de l'ordre de 1,15 [5]. Ceci conduit à une valeur de $P$ de l'ordre de $10 P_{1}$. Pour les explosifs solides, $\gamma$ est compris entre 2,5 et 3,5 [5] ; la pression d'explosion est donc beaucoup plus forte pour la même densité d'énergie.

En ce qui concerne la forme nous avons adopté une structure coaxiale de telle sorte que le morceau de conducteur cassé soit circulaire. On évite ainsi des effets de bouts qui favoriseraient la survie de l'arc et retarderaient son extinction. Quant à la largeur de la découpe, nous l'avons prise égale à $3 \mathrm{~mm}$. Cette dimension est suffisante pour assurer une technologie pas trop compliquée et assez petite pour que le disjoncteur soit rapide avec une énergie de commande acceptable [3].

2.2 Montage MÉCANiQue. - Pour être en mesure de résister aux fortes surpressions qui apparaissent lors de l'explosion du mélange détonant, les pièces qui constituent le disjoncteur sont massives. Ainsi la coupure du conducteur a lieu dans une cavité aménagée dans un bloc métallique en acier (Fig. 1). Le courant

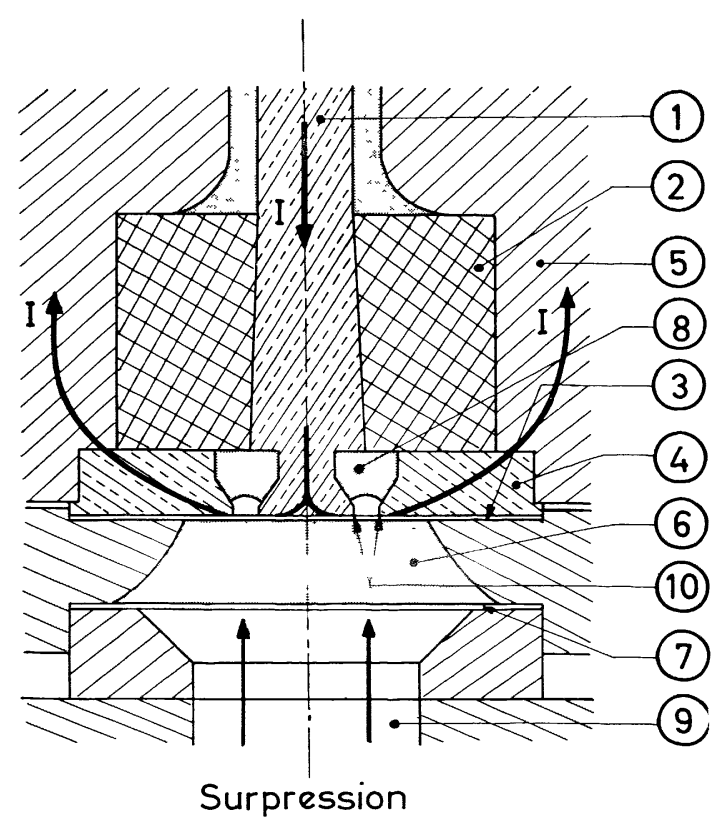

Fig. 1. - Montage mécanique du disjoncteur. arrive par une pièce de cuivre I maintenue par un isolant solide (2), passe par la plaquette de cuivre à casser (3) (diamètre $50 \mathrm{~mm}$, épaisseur $1 / 10 \mathrm{~mm}$ ) et repart par les pièces en cuivre (4) et en acier (5). La cavité (6) limitée par la plaquette (3) et un disque de cuivre recuit (7) est remplie complètement de graisse alors que le dégagement (8) ne l'est que partiellement.

Le mélange $\mathrm{H}_{2}-\mathrm{O}_{2}$, stocké dans la partie (9), est obtenu par électrolyse d'une solution de potasse contenue dans un bac solidaire du corps de l'interrupteur. La pression du gaz est estimée à partir du temps de passage d'un courant connu dans la solution de potasse. L'onde de détonation est déclenchée par l'explosion d'un fil de cuivre fin (diamètre 5/100, longueur $2 \mathrm{~mm}$ ) placé préalablement dans le mélange.

Sous l'action de la surpression, la plaquette (3) est découpée. L'anneau de découpe (de grand diamètre $16 \mathrm{~mm}$ et de petit diamètre $10 \mathrm{~mm}$ ) est projeté dans le dégagement (8); il frotte tout au long de son accélération sur les parois de la gorge de dégagement (iiD. Il continue ainsi à assurer la conduction électrique et ne débouche dans la graisse qu'après avoir acquis une grande vitesse. Le courant est alors transféré sur le fusible fixé extérieurement entre les pièces métalliques (1) et (5).

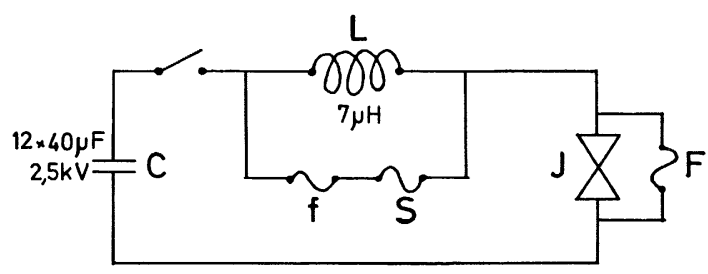

FIG. 2. - Schéma du circuit électrique. Le banc de condensateur se décharge à travers une self de $7 \mu \mathrm{H}$ et le disjoncteur étudié $\mathrm{J}$. L'explosion du fusible $\mathrm{f}$ commande le fonctionnement de $\mathrm{J}$. Le courant est alors transféré sur le fusible F.

2.3 Montage Électrique (Fig. 2). - Le courant que le disjoncteur aura à couper est fourni par la décharge d'un banc $\mathrm{C}$ de 12 condensateurs de $40 \mu \mathrm{F}$ chacun (tension nominale $2,5 \mathrm{kV}$ ) à travers une self de $7 \mu \mathrm{H}$. Le courant est alors une fonction pseudopériodique du temps de période $360 \mu$ s. L'explosion du fil fin amorçant la détonation est obtenue par dérivation d'une très faible partie du courant principal ; ce circuit est d'ailleurs ouvert peu de temps après l'explosion de $\mathrm{f}$ par l'explosion d'un fil convenablement adapté $\mathrm{S}$ car l'isolement au niveau de f n'est pas suffisant pour maintenir cette ouverture. Pour que le disjoncteur étudié J fonctionne au niveau de l'intensité maximum de courant, qui est de $16 \mathrm{kA}$ pour une tension de $2,5 \mathrm{kV}$, f est placé à une distance d'environ $20 \mathrm{~cm}$ de la plaquette de cuivre (la vitesse de propagation de l'onde de détonation est de $2800 \mathrm{~m} / \mathrm{s}$ [7]). L'ouverture de $\mathrm{J}$ transfère le courant sur le fil $F$ qui explose juste après. Dans ces essais, aucune charge n'était placée en parallèle de F ; ceci permet de tester de façon plus sévère les qualités de réisolement de $\mathrm{J}$ puisque la tension qui apparaît 
dans ces conditions est plus élevée que s'il y avait une charge.

Des sondes à débit interne [8] nous ont permis de mesurer le courant et sa dérivée temporelle dans le disjoncteur $\mathrm{J}$, dans le fil $\mathrm{F}$ et dans le circuit global.

3. Caractéristiques de l'onde de pression. - Le calcul des pressions régnant derrière l'onde de détonation s'effectue facilement. On trouve que [7,9] (*), pour une pression initiale $P_{1}$ :

- La pression des gaz brûlés juste derrière l'onde de détonation vaut :

$$
P_{2}=2(\gamma-1) K P_{1}=21 P_{1} \text {. }
$$

- La pression entre le front et la queue de l'onde passe progressivement de la valeur $21 P_{1}$ à la valeur 7,5 $P_{1}$ (cf. Fig. 3), ces deux points extrêmes se déplaçant respectivement aux vitesses $D=2800 \mathrm{~m} / \mathrm{s}$ et $D / 2$.

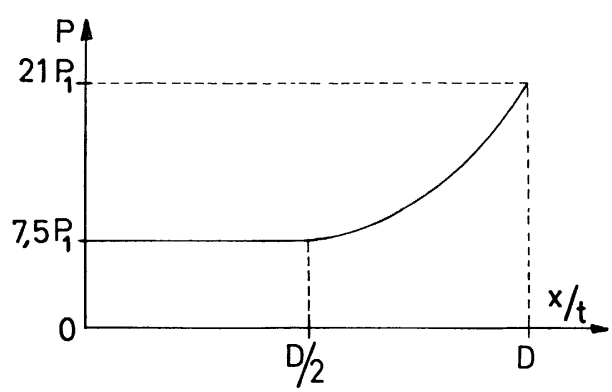

FIG. 3. - Variations de la pression d'une onde de détonation du mélange $2 \mathrm{H}_{2}+\mathrm{O}_{2}$. La vitesse de propagation du front d'onde est $D=2800 \mathrm{~m} / \mathrm{s}$.

Ainsi lors d'un choc sur une paroi rigide, la surpression engendrée par l'onde de détonation a une durée d'application limitée. Cependant, dès que nous nous plaçons à une distance assez grande du point d'initiation de la détonation (quelques dizaines de centimètres), ce temps d'application est grand par rapport au temps de coupure souhaité (une centaine de microsecondes comparée à quelques microsecondes). Aussi, nous pouvons supposer que la décroissance de la pression qui suit l'onde de détonation au cours de la coupure du conducteur est négligeable. Dans ce cas, la pression $P_{3}$ des gaz brûlés après réflexion sur la graisse de l'onde de détonation de pression $P_{2}$ vaut :

$$
P_{3}=55 P_{1} \text {. }
$$

La pression dans la graisse derrière l'onde de choc transmise vaut aussi $P_{3}$, c'est-à-dire 2200 bar pour les expériences typiques où nous avions $P_{1}=40$ bar.

- Enfin, la pression dans l'onde transmise dans la plaquette de cuivre (3) est de 4300 bar lorsque $P_{1}=40$ bar. On peut aussi montrer (*) que l'anneau de découpe parcourt alors $0,1 \mathrm{~mm}$ c'est-à-dire son épaisseur en environ $1 \mu \mathrm{s}$.

Nous voyons donc que les ordres de grandeur prévus sont parfaitement adaptés au problème de l'ouverture rapide du circuit électrique.

4. Coupure de courant. - La figure 4 représente la forme typique d'un oscillogramme obtenu lors de la coupure du courant délivré par le banc de condensateurs. Le courant $I$ circule initialement dans le seul disjoncteur $\mathrm{J}$ à l'exclusion de $\mathrm{F}$. Au moment où la plaquette de cuivre est cassée, un arc apparaît et le courant de $10 \mathrm{kA}$ est transféré sur $\mathrm{F}$ en $4 \mu$ s (Fig. $4 a$ ). Le fil explose ensuite, donnant lieu à une pointe de tension de $20 \mathrm{kV}$ pendant $1 \mu \mathrm{s}$ (Fig. 5) (la mesure de cette tension s'obtient à partir de celle de la dérivée temporelle $\mathrm{d} I / \mathrm{d} t$ et de l'inductance du circuit). Ici, F était en fait constitué de 2 fils de cuivre de $2 / 10 \mathrm{~mm}$ de diamètre et de $5 \mathrm{~cm}$ de long mis en parallèle et placés entre 2 bandes de ruban isolant électrique en vinyle (scotch, référence « $3 \mathrm{M} » 33$ ). Dans cette expérience les fils ne sont pas parfaitement adaptés et le courant principal devient très rapidement faible mais s'annule complètement en un temps beaucoup plus long (cf. Fig. 4b) ; aussi le transfert d'énergie sur une charge inductive se ferait en un temps de l'ordre de la $\mu$ s. Nous avons aussi effectué des expériences où le courant principal était coupé complètement en un temps plus long; dans ce cas, $F$ était constitué de deux fils de cuivre de $0,2 \mathrm{~mm}$ de diamètre et de $10 \mathrm{~cm}$ de long.

(*) Lucidarme, J., Legentil, M., communication privée.

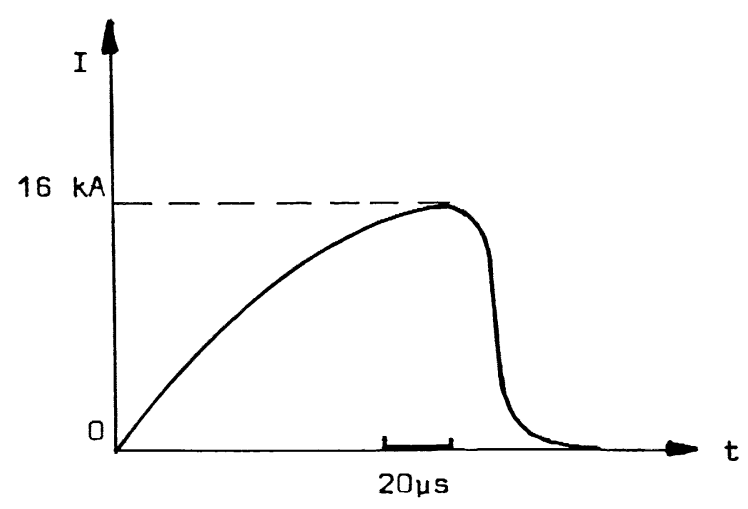

(b)

FIG. 4. - Variations du courant $I_{\mathrm{J}}$ dans le disjoncteur et du courant principal $I$ en fonction du temps. 


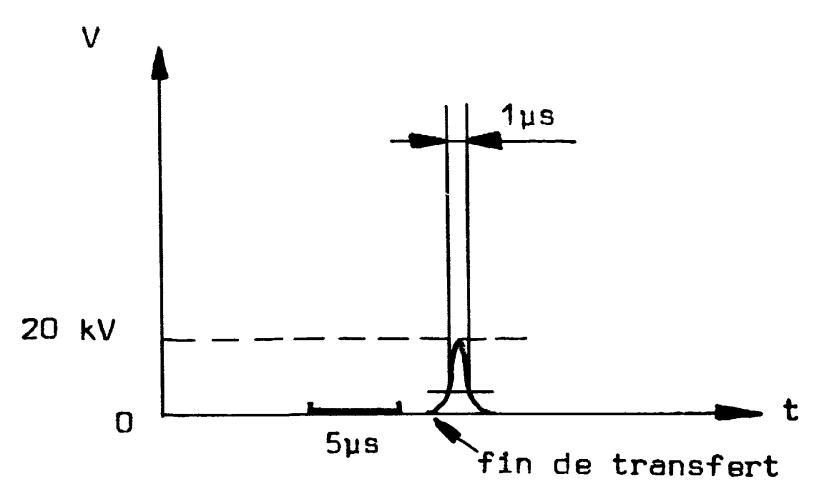

Fig. 5. - Variation de la tension $V$ aux bornes du disjoncteur en fonction du temps.

Dans toutes les expériences effectuées, la section des fils était adaptée de telle sorte que le temps de latence, c'est-à-dire le temps pendant lequel la température du fil s'élève peu, soit à pəine supérieur au temps de transfert. C'est ainsi que l'on obtient la pointe de tension la plus courte possible; sa durée est alors en général égale à environ $1 / 3 \mathrm{du}$ temps de transfert (elle peut descendre à $1 / 10$ dans certaines conditions particulières [11]). Le temps de transfert lui-même est d'autant plus court que l'inductance entre $J$ et $F$ est plus faible et que la tension d'arc est plus élevée.

5. Etude de la tension d'arc. - Nous avons cherché à préciser expérimentalement l'évolution de cette tension au cours de la commutation. Comme la variation de l'impédance du fil est faible pendant le transfert du courant, nous avons pour cette étude substitué à $F$ de résistance à froid $R_{0}$ et de self $l_{0}$ une impédance fixe $R \sim R_{0}$ et $l \sim l_{0}$ (nous avons pris $R=17,2 \mathrm{~m} \Omega$ et $l=100 \mathrm{nH}$ ). Nous avons alors mesuré le courant $i(t)$ dans $\mathrm{F}$ ainsi que sa dérivée $\mathrm{d} i / \mathrm{d} t$ et en avons déduit la tension d'arc :

$$
U(r)=R i(t)+l \frac{\mathrm{d} i}{\mathrm{~d} t} .
$$

Différents essais ont été effectués au cours desquels nous avons fait varier la pression initiale $P_{1}$ du mélange détonant et l'intensité $I$ du courant transféré sur l'impédance de dérivation. La forme de la courbe $U(t)$ reste toujours la même; la figure 6 représente une courbe typique ( $P_{1}=40$ bar, $I=10200$ A) et reproduit aussi les valeurs instantanées $R i(t)$ et $l \mathrm{~d} i(t) / \mathrm{d} t$ qui nous ont permis de la tracer. Tout d'abord la tension $U$ croît jusqu'à une valeur maximum $U_{\mathrm{M}}$ atteinte au temps $t_{0}$ puis elle décroît rapidement jusqu'à la valeur $U_{0}=R I$. En ce point, atteint au bout d'un temps $t_{1}$, $\mathrm{d} i / \mathrm{d} t=0$; le transfert du courant sur la résistance étalonnée est alors terminé. Pour les valeurs de $U$ proches de $U_{\mathrm{M}}$, la quasi-totalité du courant est déjà transférée sur l'impédance de dérivation et $\mathrm{d} i / \mathrm{d} t$ passe par un maximum. Nous avons aussi tracé la courbe en représentant $U$ en fonction du courant qui traverse l'arc (Fig. 7).

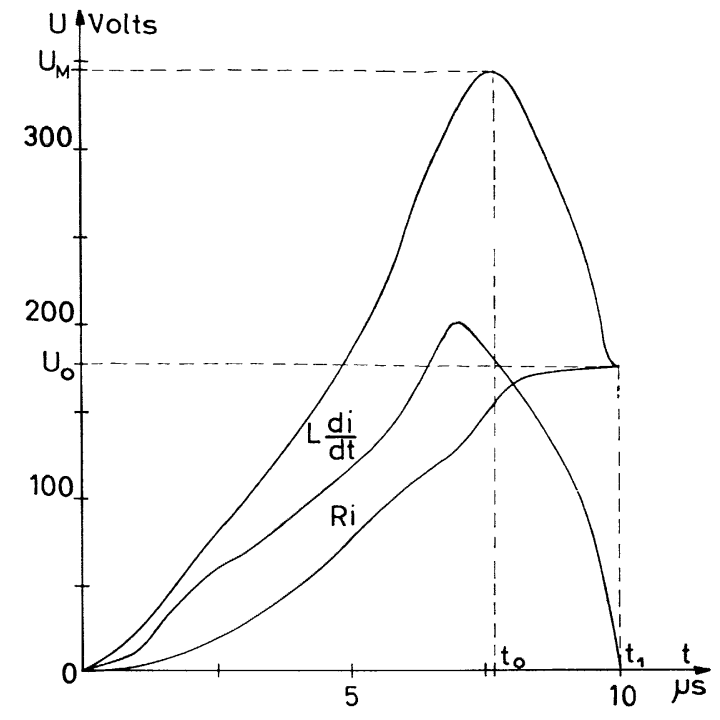

Fig. 6. - Variations de la tension d'arc en fonction du temps de transfert du courant sur une impédance $\left(P_{1}=40\right.$ bar, $I=10 \mathrm{kA})$.

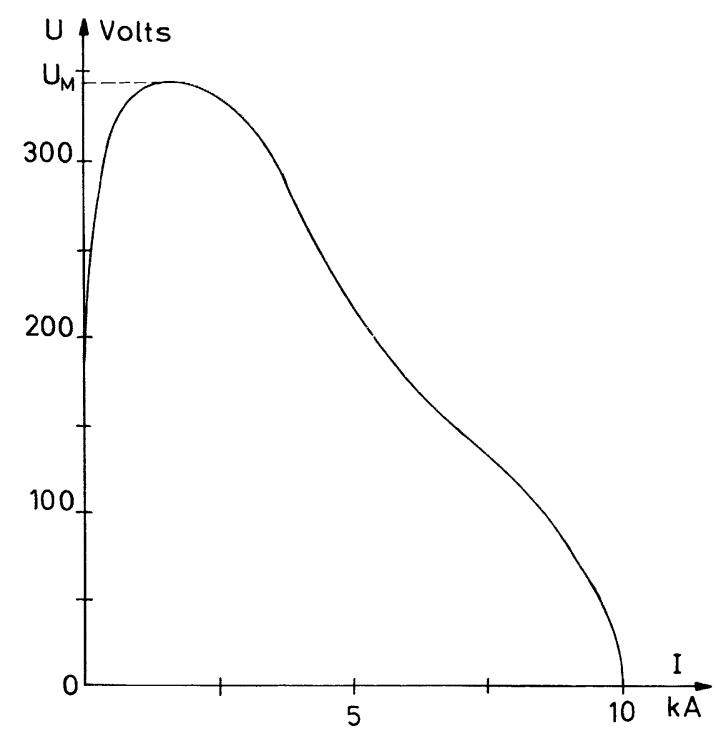

FIG. 7. - Variations de la tension d'arc en fonction du courant qui le traverse $\left(P_{1}=40 \mathrm{bar}\right)$.

La tension maximale $U_{\mathrm{M}}$ est toujours de l'ordre de quelques centaines de volts. En première approximation, à pression $P_{1}$ fixée, elle croît linéairement avec le courant $I$ de même que le temps de transfert $t_{1}$. Les variations de $U_{\mathrm{M}}$ et $t_{1}$ en fonction de $I$ pour $P_{1}$ fixé à 40 bar sont données sur la figure 8 . Nous avons constaté de plus qu'à courant transféré égal, la tension $U_{\mathrm{M}}$ est plus importante pour les pressions $P_{1}$ les plus élevées ( $I=10 \mathrm{kA}: U_{\mathrm{M}}=280 \mathrm{~V}$ pour $P_{1}=40$ bar, $U_{\mathrm{M}}=350 \mathrm{~V}$ pour $P_{1}=60$ bar).

Le disjoncteur que nous venons de décrire permet de couper en $1 \mu \mathrm{s}$ des courants de l'ordre de $10^{4} \mathrm{~A}$. Par mise en série et parallèle de telles unités, on peut donc transférer très rapidement sur une charge d'utilisation l'énergie stockée dans une bobine. Cependant, pour que l'utilisation de ce procédé soit envisageable à haut 


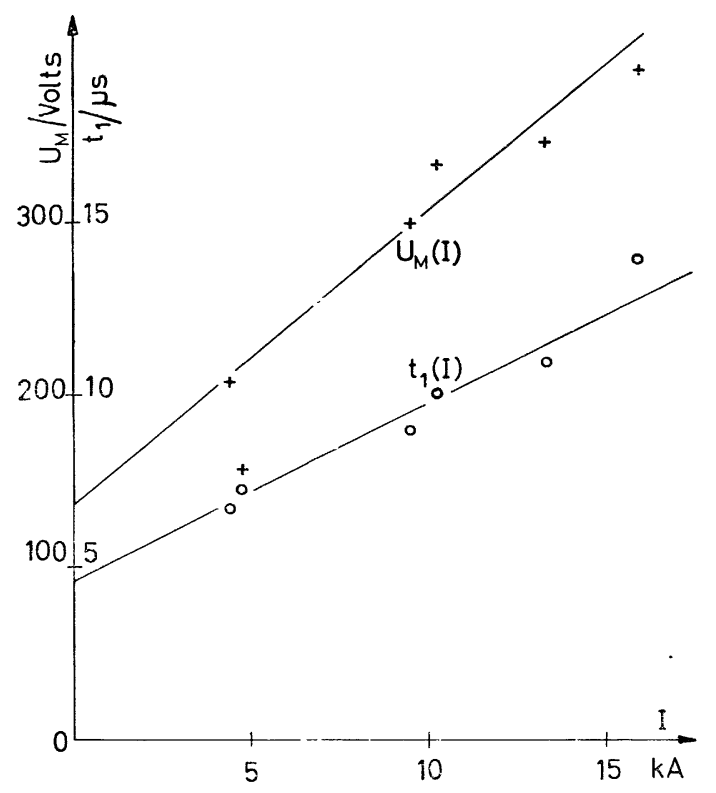

Fig. 8. - Variations de la tension maximale d'arc $U_{\mathrm{M}}$ et du temps de transfert $t_{0}$ en fonction du courant transféré $I$ $\left(P_{1}=40 \mathrm{bar}\right)$.

niveau d'énergie, il faut que l'énergie chimique mise en œuvre soit faible comparée à l'énergie transférée. C'est pourquoi nous avons cherché à déterminer l'énergie minimum nécessaire pour obtenir la coupure.

Nous avons déjà indiqué la forme de l'onde de pression (Fig. 3). Ainsi, tout au long de sa progression dans le mélange $\mathrm{H}_{2}-\mathrm{O}_{2}$, l'onde s'étale dans l'espace et acquiert de l'énergie et de la quantité de mouvement. Or, cette onde doit posséder suffisamment d'énergie pour assurer la découpe du conducteur et la mise en mouvement du morceau de conducteur cassé et de la graisse. Pour acquérir toute cette énergie, elle devra alors parcourir une certaine distance $L$ dans le mélange $\mathrm{H}_{2}-\mathrm{O}_{2}$ ou, ce qui revient au même, être initiée à une distance $L$ de la surface libre de la graisse.
Une étude expérimentale nous a montré que $L$ devait au moins être égal à $8 \mathrm{~cm}$ pour obtenir la simple découpe d'un conducteur de cuivre d'épaisseur $0,1 \mathrm{~mm}$ lorsque $P_{1}=40$ bar. En considérant que le gaz utile à la découpe se trouve dans le volume délimité par deux cylindres concentriques ayant pour section droite la couronne sous-tendue par les lèvres de la coupure du conducteur et pour hauteur $8 \mathrm{~cm}$, on trouve que l'énergie chimique utilisée est de 2,3 kJ. Or, l'onde de pression doit non seulement assurer une découpe franche du conducteur mais aussi fournir de l'énergie cinétique au morceau cassé et à la graisse qui assure l'isolement. Il faudra donc qu'elle acquière plus d'énergie que dans le cas considéré ci-dessus. Autrement dit, l'énergie chimique dépensée pour assurer une bonne commutation du courant sur le fusible devra être supérieure à $2,3 \mathrm{~kJ}$.

Cette énergie dépasse celle du banc de condensateurs utilisé dans nos expériences $(1,5 \mathrm{~kJ})$. Cependant, un conducteur d'épaisseur $1 / 30 \mathrm{~mm}$ aurait suffi à tenir le courant de $15 \mathrm{kA}$ pendant $90 \mu$ s et l'énergie chimique nécessaire n'aurait plus été que de l'ordre de $1 \mathrm{~kJ}$. Il semble toutefois que dans le meilleur des cas, énergie transférée et énergie chimique minimum mises en jeu lors de la commutation soient tout au plus du même ordre de grandeur.

6. Conclusion. - La possibilité de réaliser des disjoncteurs à rupture mécanique capable de transférer des courants de $10^{4} \mathrm{~A}$ en $1 \mu$ s a été mise en évidence. La méthode de création de la pression de commande de l'ouverture (détonation d'un mélange $\mathrm{H}_{2} \cdot \mathrm{O}_{2}$ sous pression créé par électrolyse) est très simple et peut être appliquée à de petites unités où les critères de rendement sont secondaires. Pour des transferts d'énergie au niveau du mégajoule ou plus, ces derniers deviennent prépondérants. Il est alors nécessaire de se tourner vers des techniques plus performantes.

\section{Bibliographie}

[1] Rioux-Damidau, F., Revue Phys. Appl., sous presse.

[2] Rioux, C., Revue Phys. Appl., sous presse.

[3] Rioux, C., Rioux-Damidau, F., Revue Phys. Appl. 9 (1974) 539.

[4] Grossetete, F., Rioux, C., C. R. Hebd. Séan. Acad. Sci. B 270 (1970) 1384.

[5] Lange Handbook of Chemistry, p. 1516.

[6] Kaziz, A., Hurwitz, H. and Sternberg, H. M., Phys. Fluids 4 (1961) 380.
[7] Landau, L. et Lifchitz, E., Mécanique des Fluides, Moscou 1971 (Chap. XIV).

[8] Rioux, C., Rioux, F., Revue Phys. Appl. 7 (1972) 303 et 313.

[9] Delmas, A., Thèse $3^{\mathrm{e}}$ cycle, Paris (1972).

[10] Zeldovitch, B., Kogarkd, S. M., Simonov, N. N., Zh. Tekh. Fiz. 26 (1956) Traduction anglaise.

[11] Antoni, B., Revue Phys. Appl. 9 (1974) 331. 